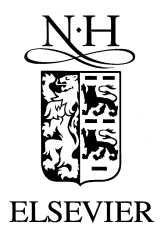

15 April 1999

\title{
Vortex evolution in parametric wave mixing
}

\author{
Dmitri V. Petrov ${ }^{1}$, Gabriel Molina-Terriza, Lluis Torner \\ Laboratory of Photonics, Department of Signal Theory and Communications, Universitat Politecnica de Catalunya, \\ Gran Capitan UPC-D3, ES 08034 Barcelona, Spain
}

Received 16 December 1998; received in revised form 10 February 1999; accepted 11 February 1999

\begin{abstract}
We investigate the evolution of vortex wave front dislocations in multiple-wave second-harmonic generation processes in quadratic nonlinear media. Vortices nested in finite-size host beams are shown to nucleate and to annihilate in pairs, and to move across the transverse wave front during the beam evolution. A closed-form model that holds under conditions of negligible-depletion of the pump beam is developed to describe the vortex dynamics in order to predict the number of vortices present in the wave fronts of the beams at any instance of the propagation. Results are compared with numerical simulations of the full governing equations and with experimental observations. Limitations of the model are outlined. (c) 1999 Elsevier Science B.V. All rights reserved.
\end{abstract}

\section{Introduction}

Singular beams, namely those that contain wave front dislocations [1,2], exhibit unusual properties even in linear optics, in free-space propagation. Screw dislocations, or optical vortices, are a common dislocation type [3-5]. They are spiral phase-ramps around a phase singularity where the phase of the wave is undefined thus its amplitude must vanish. The order of the singularity multiplied by its sign is referred to as the topological charge of the vortex.

In the case of ideal dark-like fields that extent to infinity in the transverse direction, the net charge of the existing dislocations is conserved during the wave propagation in a linear continuous medium. However, it has been shown recently both experimentally and theoretically by Soskin and co-workers that such is not necessarily the case with finite-size beams [6]. In particular, they showed that in a combined beam, made of the superposition of a vorticity-less Gaussian beam and a Gaussian envelope beam with vortices nested (so-called singular beam), vor-

\footnotetext{
${ }^{1}$ Current address: Department of Fundamental Chemistry, University Federal of Pernambuco, Recife, CEP50740-540, Brazil.
}

tices can nucleate, annihilate, or move from or to the beam tails during free propagation in a linear medium. Briefly, the main results are: Addition of a coherent coaxial vortex-free beam to a beam with a $m$-charged vortex may change the number of vortices and the net topological charge of the dislocations present in the combined beam. Depending on the parameters of the two beams, the number of vortices in the combined beam may vary between 0 , $|m|$ and $2|m|$. The net topological charge of all the vortices present is either $m$ or 0 . More precisely, in the free-space propagation of the combined beam from the waist of the singular beam to infinity, the number of vortices present in the combined beam changes for any choice of parameters of the input light, with the exception of the case of exactly equal transverse sizes of the singular and the vortex-free input beams.

Parametric, nonlinear mixing of multiple waves containing wave front dislocations can potentially offer a much richer variety of phenomena. Because of the parametric interaction, the waves exchange not only energy with each other but also nonlinear phase-shift, hence wave fronts. Therefore, a general question arises about the evolution of the topological charges existing in the waves of different frequencies. Such evolution, including the number of dislocations present, depends on the competition 
between the various features of the interacting waves. Here we consider a particular but important case of secondharmonic generation of light in quadratic nonlinear media, where one fundamental wave at frequency $\omega$ and one second-harmonic wave at frequency $2 \omega$ parametrically $\operatorname{mix}$.

In up-conversion of fundamental waves with moderate input powers and wide beams, light undergoes frequency doubling together with the generation of a phase dislocation nested in the second harmonic beam [7-10]. The topological charge of the dislocation in the secondharmonic beam is dictated by the charge of the input light. A totally different situation is encountered when a coherent second-harmonic wave, with a well defined topological structure, is also input at the crystal together with the fundamental beam. Here we analyze the case that previously was studied by us experimentally [11], namely, when the input fundamental is a Gaussian beam that contains a single charge vortex and the input second-harmonic seed beam is a vorticity-less Gaussian beam.

First, we develop an analytical model of the vortex dynamics based on the assumption of negligible-depletion of the fundamental beam. It is shown that under such regime the vortex dynamics can be described with an approach similar to that in Ref. [6]. Then, using that model we predict a rich dynamics of spontaneous appearance and disappearance of vortices during the beam evolution. Results are compared with numerical simulations of the full governing equations and compared with experimental observations. Limitations of the model due to the failure of the negligible pump-depletion regime are outlined.

\section{Analytical model for negligible pump-depletion}

The evolution equations for the slowly-varying normalized envelopes of the light waves in type I phase-matching second-harmonic generation geometries in the absence of Poynting vector walk-off can be written as

$i \frac{\partial a_{1}}{\partial \xi}-\frac{\alpha_{1}}{2} \nabla_{\perp}^{2} a_{1}+a_{1}^{*} a_{2} \exp (-i \beta \xi)=0$,

$i \frac{\partial a_{2}}{\partial \xi}-\frac{\alpha_{2}}{2} \nabla_{\perp}^{2} a_{2}+a_{1}^{2} \exp (i \beta \xi)=0$,

where $a_{1}$ is the normalized amplitude of the fundamental frequency (FF) wave, $a_{2}$ is the normalized amplitude of the second-harmonic (SH) wave, $\alpha_{1}=-1$ and $\alpha_{2}=$ $-k_{1} / k_{2}$. Here $k_{\nu}$, with $\nu=1,2$, are the linear wave numbers of the waves. In practice $\alpha_{2} \simeq-0.5$, so in the calculations we set $\alpha_{2}=-0.5$. The transverse coordinates are normalized to the beam width $\eta$, and the propagation coordinate $\xi$ is normalized to twice the diffraction length of the fundamental beam $l_{d 1}=k_{1} \eta^{2} / 2$. The parameter $\beta$ is given by $\beta=k_{1} \eta^{2} \Delta k$, where $\Delta k=2 k_{1}-k_{2}$ is the wave vector mismatch.
The linear parts of the above equations are the scalar wave equations at the fundamental and second-harmonic frequencies in the paraxial approximation. One readily finds that they have solutions consisting of a Gaussian envelope carrying a nested screw phase dislocation with topological charge $m$, with the form

$$
\begin{aligned}
\Psi= & \left(\frac{r}{w_{0}}\right)^{|m|}\left(\frac{1}{1-i 2 \alpha_{\nu} \xi / w_{0}^{2}}\right)^{|m|+1} \\
& \times \exp \left[-\frac{r^{2}}{w_{0}^{2}\left(1-i 2 \alpha_{\nu} \xi / w_{0}^{2}\right)}\right] \exp (i m \phi),
\end{aligned}
$$

where $w_{0}$ is the corresponding beam waist.

Let the input field consist of a beam of FF with a single charge vortex, i.e.,

$$
\begin{aligned}
a_{1}(r, \xi)= & A_{0} \frac{r}{w_{0 v}} \frac{1}{\left(1+i \xi_{v}\right)^{2}} \\
& \times \exp \left[-\frac{r^{2}}{w_{0 v}^{2}\left(1+i \xi_{v}\right)}\right] \exp (i \phi),
\end{aligned}
$$

and a seed of $\mathrm{SH}$ without phase dislocation, given by

$A_{2}^{s}(r, \xi)=A_{0}^{s}\left(\frac{1}{1+i \xi_{s}}\right) \exp \left[-\frac{r^{2}}{w_{0 s}^{2}\left(1+i \xi_{s}\right)}\right]$.

Here $\xi_{v}=\xi / z_{0 v}$, with $z_{0 v}=w_{0 v}^{2} /\left(2\left|\alpha_{1}\right|\right), w_{0 v}$ is the waist of fundamental beam, $A_{0}$ is the FF input amplitude, $\xi_{s}=\xi / z_{0 s}$, with $z_{0 s}=w_{0 s}^{2} /\left(2\left|\alpha_{2}\right|\right), w_{0 s}$ is the waist of the $\mathrm{SH}$ seed, and $A_{0}^{s}$ is the input amplitude of the $\mathrm{SH}$ seed. Notice a change of sign in expressions (3), (4) relative to (2) that is due to the fact that the $\alpha_{\nu}$ are always negative numbers.

The evolution equation for the slowly-varying field envelope of the second-harmonic beam $a_{2}(r, \xi)$ at negligible pump-depletion regime, defined as the regime where

$\int\left|a_{1}(r, \xi)\right|^{2} \mathrm{~d} r=$ const,

is given by

$$
i \frac{\partial a_{2}(r, \xi)}{\partial \xi}-\frac{\alpha_{2}}{2} \nabla_{\perp}^{2} a_{2}(r, \xi)=-a_{1}^{2}(r, \xi) \exp (i \beta \xi) \text {. }
$$

A trial solution of (6) is

$$
\begin{aligned}
A_{2}^{p}(r, \xi)= & A_{2}(\xi)\left(\frac{r}{w_{0 v}}\right)^{2}\left(\frac{1}{1+i \xi_{v}}\right)^{3} \\
& \times \exp \left[-2 \frac{r^{2}}{w_{0 v}^{2}\left(1+i \xi_{v}\right)}\right] \exp (i 2 \phi),
\end{aligned}
$$


which corresponds to a double-charge vortex nested in a Gaussian-like beam with the same confocal parameter as a fundamental beam [9]; also its radial dependence is identical to that of the source term. Using (7) in (6), one arrives at

$$
\begin{aligned}
& i \frac{\mathrm{d} A_{2}(\xi)}{\mathrm{d} \xi}-\left[\frac{6}{w_{0 v}^{2}\left(1+i \xi_{v}\right)}-\frac{4 r^{2}}{w_{0 v}^{4}\left(1+i \xi_{v}\right)^{2}}\right] \\
& \quad \times\left(\alpha_{1}-2 \alpha_{2}\right) A_{2}(\xi)=-A_{0}^{2} \frac{1}{1+i \xi_{v}} \exp (i \beta \xi) .
\end{aligned}
$$

Thus, letting $\alpha_{1}=-1$ and $\alpha_{2}=-1 / 2$, yields

$i \frac{\mathrm{d} A_{2}(\xi)}{\mathrm{d} \xi}=-\frac{1}{1+i \xi_{v}} A_{0}^{2} \exp (i \beta \xi)$.

This equation can be integrated directly to obtain

$$
A_{2}(\xi)=i A_{0}^{2} z_{0 v} f\left(\xi_{v}, \beta_{v}\right) \text {, }
$$

where $\beta_{v}=\beta z_{0 v}$, and

$f\left(\xi_{v}, \beta_{v}\right)=\int_{0}^{\xi_{v}} \frac{\exp \left(i \beta_{v} y\right)}{1+i y} \mathrm{~d} y$.

Therefore, the generated second harmonic field is

$$
\begin{aligned}
A_{2}^{p}(r, \xi)= & i A_{0}^{2} z_{0 v} f\left(\xi_{v}, \beta_{v}\right)\left(\frac{r}{w_{0 v}}\right)^{2}\left(\frac{1}{1+i \xi_{v}}\right)^{3} \\
& \times \exp \left[-2 \frac{r^{2}}{w_{0 v}^{2}\left(1+i \xi_{v}\right)}\right] \exp (i 2 \phi) .
\end{aligned}
$$

The whole SH field $a_{2}$ is the superposition of the generated field $A_{2}^{p}$ given by (12) and the seed $A_{2}^{s}$ given by (4).

To find the positions of the vortices in the total $\mathrm{SH}$ beam we need to write two equations, one giving the radius of the zero-amplitude points where the modulus of the amplitudes of the Gaussian seed and the generated second harmonic wave are equal to each other, and another giving the angular coordinate where the destructive interference between both beams occurs [4]. The first equation is

$$
\left|A_{2}^{p}(r, \xi)\right|=\left|A_{2}^{s}(r, \xi)\right|,
$$

Fig. 1. Predicted number of single-charge vortex phase-dislocations present in the wave front of the SH beam using the model of negligible depletion of the FF beam. (a) $\kappa=0.5$, (b) $\kappa=1$, (c) $\kappa=2$. The dashed lines appearing in (b) correspond to $\beta_{v}=10$. Solid lines in all plots correspond to $\beta_{v}=0$. Crosses indicate the location of the simulations shown in Figs. 2 and 4, triangles correspond to Figs. 3, squares correspond to Fig. 5, and circles correspond to Fig. 6. The numbers $m$ and $n$ in the labels $[m ; n]$ stand for the number of single charge vortices present and for their total topological charge, respectively. and gives the number of possible amplitude zeros in the total beam. The second equation is

$\Phi_{2}^{p}(r, \xi)=\Phi_{2}^{s}(r, \xi) \pm \pi$,

and gives the azimuthal location of the vortices present. In what follows we will only make use of Eq. (13). Using

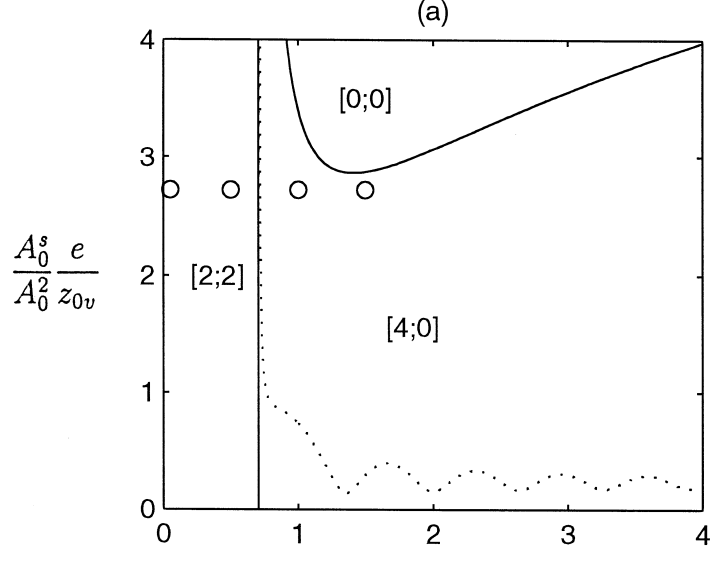

(b)

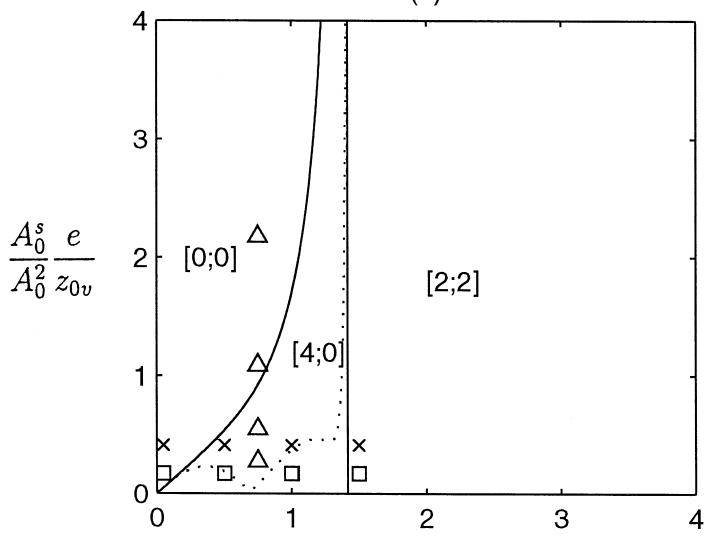

(c)

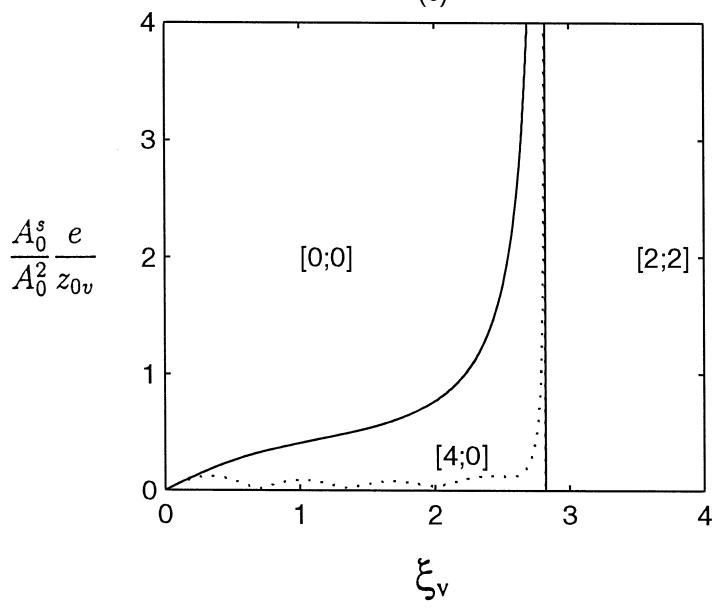


definitions similar to those in Ref. [6] for easy comparison, Eq. (13) can be explicitly written as

$\rho^{2}=\frac{A_{0}^{s}}{A_{0}^{2}} \frac{1}{z_{0 v}} \frac{1}{\left|f\left(\xi_{v}, \beta_{v}\right)\right|} C\left(\xi_{v}, \kappa\right) \exp \left[\alpha\left(\xi_{v}, \kappa\right) \rho^{2}\right]$,

where $\rho=r / w_{0 v}$,

$\kappa=\frac{w_{0 s}}{w_{0 v}}$

and

$\alpha\left(\xi_{v}, \kappa\right)=\frac{2}{1+\xi_{v}^{2}}-\frac{1}{\kappa^{2}+\xi_{v}^{2} /\left(4 \kappa^{2}\right)}$,

$$
C\left(\xi_{v}, \kappa\right)=\frac{\left(1+\xi_{v}^{2}\right)^{3 / 2}}{\left[1+\xi_{v}^{2} /\left(4 \kappa^{4}\right)\right]^{1 / 2}} .
$$

Depending on the values of the various parameters of the two Gaussian envelopes of the FF pump and the SH seed, we identified three possible scenarios:

A: $\alpha\left(\xi_{v}, \kappa\right)<0$. Then, Eq. (15) has only one root two times degenerate. This corresponds to the presence of two vortices in the total SH beam which are located at the same radial coordinate but at a different symmetrical azimuthal position, and occurs when:

1. $\kappa>1 / \sqrt{2}$, for propagation distances $\xi_{v}>\sqrt{2} \kappa$,

2. $\kappa<1 / \sqrt{2}$, for propagation distances $\xi_{v}<\sqrt{2} \kappa$.

B: $\alpha\left(\xi_{v}, \kappa\right)=0$. Eq. (15) has only one, double-degenerate root. This corresponds again to the presence of two single-charge vortices in the $\mathrm{SH}$ beam, and occurs either when $\kappa=1 / \sqrt{2}$ for all values of $\xi_{v}$, or when $\xi_{v}=\sqrt{2} \kappa$ for all values of $\kappa$.

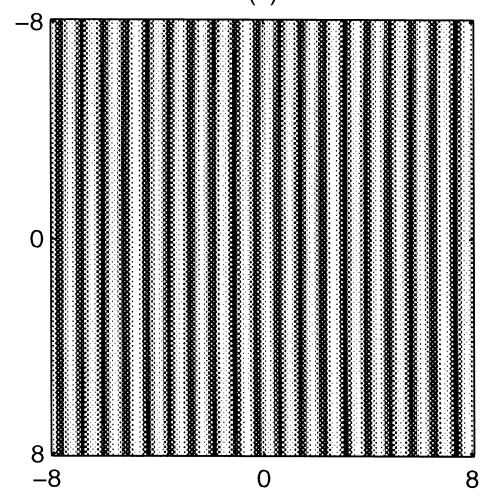

(c)

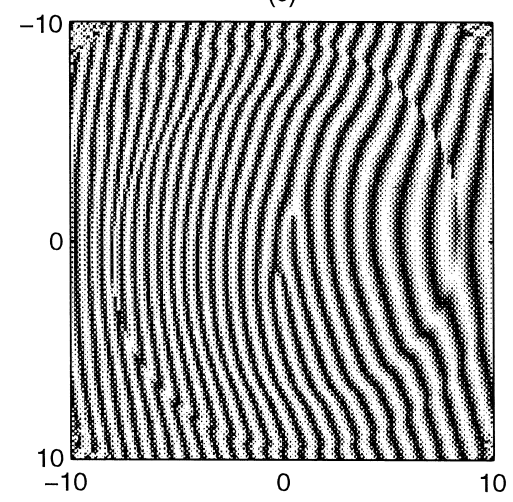

(b)

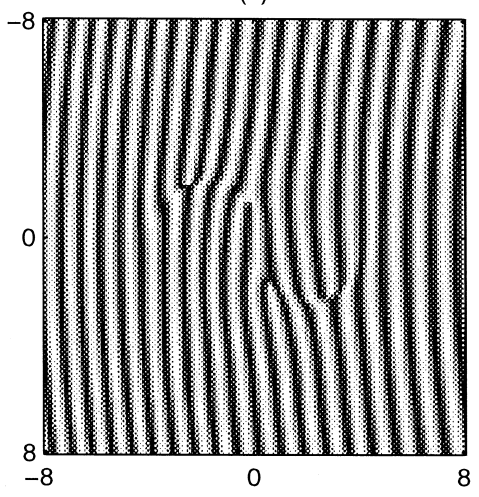

(d)

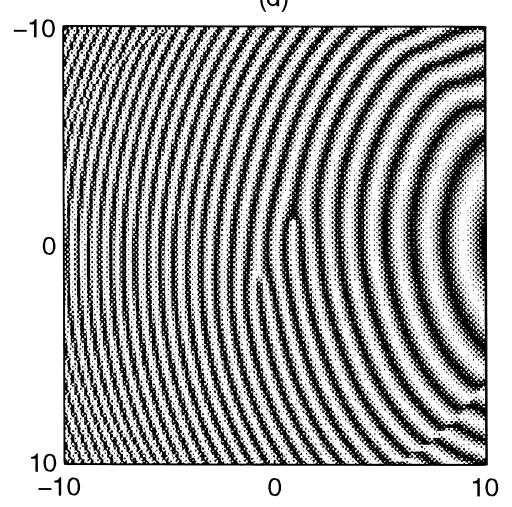

Fig. 2. Numerically obtained interferograms for the SH beam at different propagation distances inside the nonlinear crystal. Input conditions are: FF pump peak amplitude $A_{0}=0.5$, SH seed peak amplitude $A_{0}^{s}=0.075$, width of the pump $w_{0 v}=2$, width of the seed $w_{o s}=2$, thus $\kappa=1$, and exact phase-matching $(\beta=0$ ). Propagation distances: (a) $\xi=0.1$, (b) $\xi=1$, (c) $\xi=2$, (d) $\xi=3$. Here and in all plots, when comparing with Fig. 1 recall that $\xi_{v}=2 \xi / w_{0 v}^{2}$. 
C: $\alpha\left(\xi_{v}, \kappa\right)>0$. In this case there are three possibilities, as in the case of linear propagation [6]. Namely,

1. No roots of Eq. (15). Thus, no vortices in the total $\mathrm{SH}$ beam. This occurs when

$$
\frac{A_{0}^{s}}{A_{0}^{2}} \frac{e}{z_{0 v}}>\frac{f\left(\xi_{v}, \beta_{v}\right)}{C\left(\xi_{v}, \kappa\right) \alpha\left(\xi_{v}, \kappa\right)},
$$

where $e=\exp (1)$.

2. Two double-degenerate roots of Eq. (15). This corresponds to four single-charge vortices in the $\mathrm{SH}$ beam, and occurs when

$$
\frac{A_{0}^{s}}{A_{0}^{2}} \frac{e}{z_{0 v}}<\frac{f\left(\xi_{v}, \beta_{v}\right)}{C\left(\xi_{v}, \kappa\right) \alpha\left(\xi_{v}, \kappa\right)} .
$$

3. One double-degenerate root of Eq. (15). This corresponds to the critical points where nucleation or annihilation of two vortex twin pairs takes place, and occurs when

$$
\frac{A_{0}^{s}}{A_{0}^{2}} \frac{e}{z_{0 v}}=\frac{f\left(\xi_{v}, \beta_{v}\right)}{C\left(\xi_{v}, \kappa\right) \alpha\left(\xi_{v}, \kappa\right)}
$$

In Fig. 1 we have plotted the summary of all such possibilities for $\beta=0$ and $\beta \neq 0$. The labels of the differ-

(a)

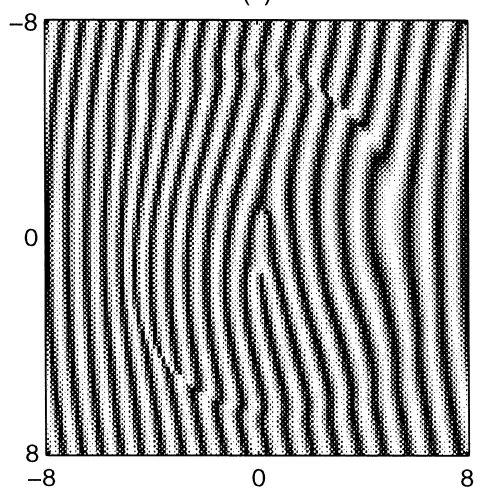

(c)

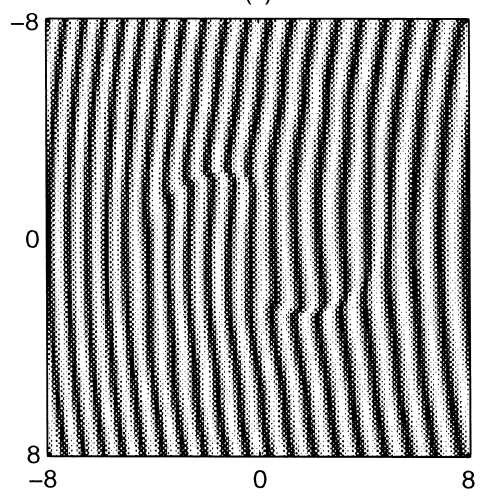

ent regions of the plot stand for the predicted total number $n$ of single-charge vortices present in the total $\mathrm{SH}$ beam as a function of propagation distance inside the nonlinear crystal, assuming negligible pump-depletion conditions, for three values of ratio between the width of the two input FF pump and $\mathrm{SH}$ seed beams $\kappa$. The discrete points appearing in the figure correspond to some of the conditions of the numerical simulations showed below and are included here for easier reading.

The solid vertical lines located at $\xi_{v}=\sqrt{2} \kappa$ separate the diagram into two parts. When $\kappa<1 / \sqrt{2}$, in the left-hand side of the diagram the number of vortices is 2 , whereas in the right-hand side the number of vortices may be 0 or 4 , depending on the amplitudes of the FF pump and $\mathrm{SH}$ seed beams. In the latter case, there are 2 vortices whose charge has the same sign as the charges of the input FF beam, hereafter referred to as positive charges, and 2 vortices whose charge has the opposite sign as the charges of the input FF beam, hereafter referred to as negative charges. Hence, total charge zero. The complementary scenario is obtained when $\kappa>1 / \sqrt{2}$. The solid curved line appearing in the diagram holds for the case of exact phase-matching $(\beta=0)$ and corresponds to the domain

(b)

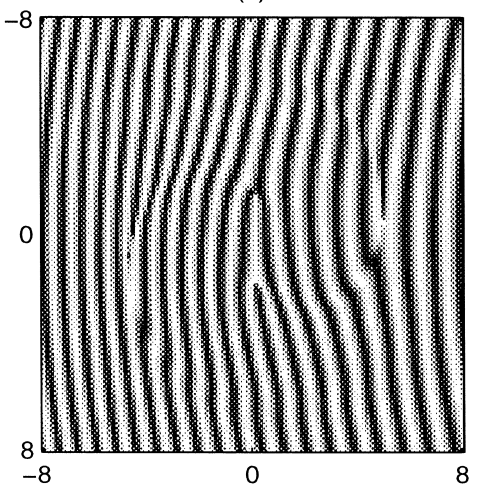

(d)

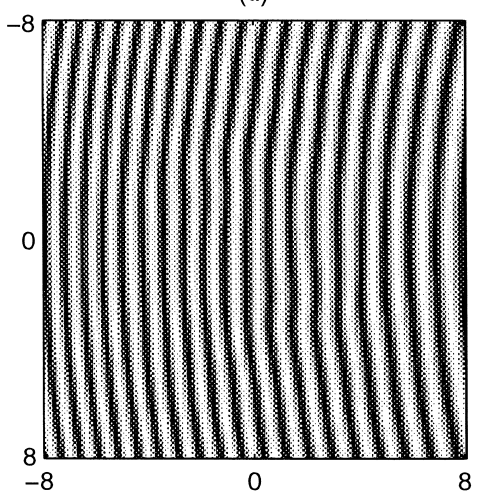

Fig. 3. Numerically obtained interferograms for the SH beam at a fixed propagation distance, for different values of the SH seed peak amplitude. Input conditions identical to those in Fig. 2, but here the input SH seed peak amplitude is: (a) $A_{0}^{s}=0.05$, (b) $A_{0}^{s}=0.1$, (c) $A_{0}^{s}=0.2$, (d) $A_{0}^{s}=0.4$. Propagation distance: $\xi=1.5$. 
boundary defined by Eq. (20). Above that curve there are no vortices in the SH beam. The effect of a finite mismatch on the location of that curve is shown in the plot with the dashed lines. One observes that when $\beta \neq 0$ the area of the region where the $\mathrm{SH}$ beam contains 4 vortices is much smaller that at exact phase-matching.

Notice that features in Fig. 1 are qualitatively similar to those that are obtained in linear media [6]. The quantitative differences are due to the dynamics of the $\mathrm{SH}$ generation process. In particular, in the case we examine here the critical value of $\kappa$ separating different regimes of vortex dynamics is $\kappa=1 / \sqrt{2}$, to be compared with the value $\kappa=1$ that holds in the linear case. Such difference is due to the fact that under low-depletion conditions the generated $\mathrm{SH}$ beam is $\sqrt{2}$ times narrower than the FF pump beam.

One of the most important results shown by Fig. 1 is that the aspect ratio $\kappa$ is also the parameter that determines the value of the total topological charge of the vortices present in the $\mathrm{SH}$ beam at large propagation distances. For $\kappa>1 / \sqrt{2}$ such net charge is always 2, whereas when $\kappa<1 / \sqrt{2}$ the net charge is always 0: there are either no vortices at all in the wave front of the $\mathrm{SH}$ beam, or there are 2 vortex-pairs with zero net charge.

\section{Numerical simulations}

To check the predictions and limitations of the negligible pump-depletion model, not only in terms of the number of vortices predicted by (13) and shown in Fig. 1 but also in terms of their azimuthal location predicted by (14) but not visible in Fig. 1, we performed series of simulations by solving the full governing equations (1) with a split-step Fourier algorithm for a variety input conditions in the negligible pump-depletion regime. We examined the evolution of input beams with the form $a_{1}(\xi=0)=$ $A_{0}\left(r / w_{0 v}\right) \exp \left[-\left(r / w_{0 v}\right)^{2}\right] \exp (i \phi)$ and $a_{2}(\xi=0)=A_{0}^{s}$ $\exp \left[-\left(r / w_{0 s}\right)^{2}\right]$. The accuracy of the negligible-depletion approximation was examined by monitoring the evolution of (5) along propagation distance. To analyze the presence of wave front dislocations we monitored the interference pattern arising by superposing the evolving beams with a reference plane wave titled slightly relative to the propaga- (a)

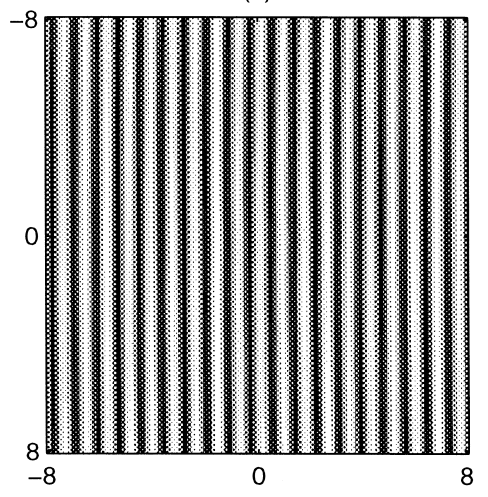

(c)

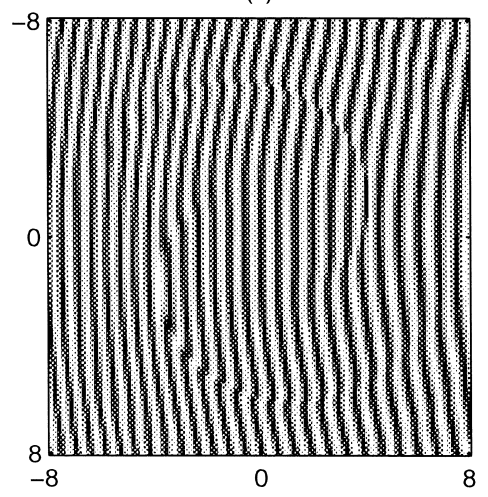

(b)

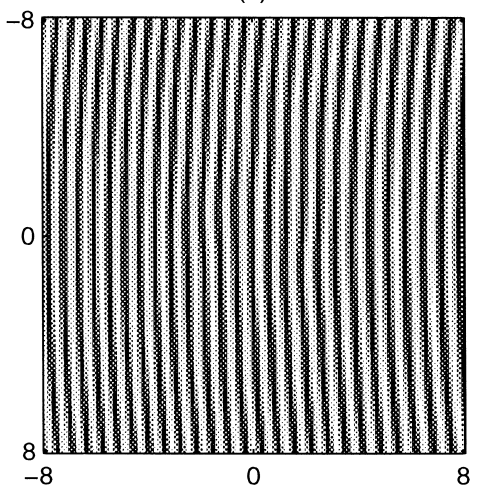

(d)

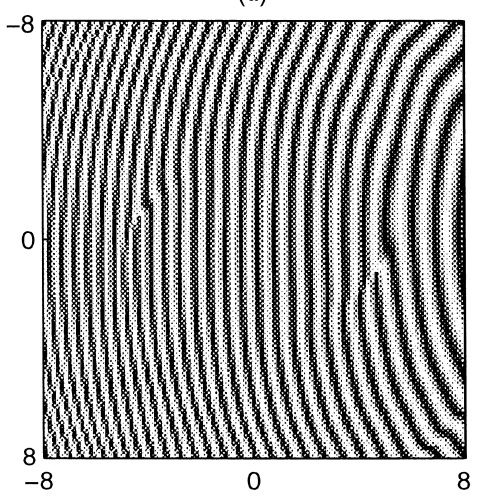

Fig. 4. Numerically obtained interferograms for the SH beam obtained with identical input conditions as in Fig. 2, but for the normalized wave vector mismatch $\beta=5$ (i.e., $\beta_{v}=10$ ). Propagation distances as in Fig. 2. 
tion axis. The resulting scaled wave amplitude exhibits a characteristic fork at the position of the wave-front dislocation and its topological charge is visually evaluated [12]. Nearby the critical propagation distance $\xi_{v}=\sqrt{2} \kappa$, Fig. 1 predicts the existence of vortices very far from the beam center that come from, or go to infinity. In the numerical simulations for such conditions the corresponding vortices would be located either outside the calculation window or at points where the field amplitude amounts to very small values, comparable to the accuracy of the numerical scheme. Under such conditions, the outcome of the numerical experiments is not conclusive. Thus, in what follows we do not consider such cases.

Fig. 2 shows the interference patterns for the secondharmonic beam monitored at different propagation distances $\xi_{v}$ of a typical example where the low-depletion conditions are fulfilled. The location of the corresponding input conditions and propagation distances in Fig. 1(b) is indicated with crosses. The fundamental beam is observed to carry only a single charge dislocation, as expected from the negligible pump-depletion approximation, thus it is not shown. As predicted by Fig. 1(b), no vortices are observable in the numerical interferogram at small propagation distances. When the propagation distance increases two pairs of dislocations with opposite charges appear. The vortices with positive charges remain nearby the propagation axis and their radial position does not change considerably with propagation distance. Their angular positions do change, as the charges rotate. On the contrary, the vortices with negative charges move away from the center, and they eventually disappear in the beam tails. Beyond $\xi_{v}=\sqrt{2}$ only two positive vortices are visible in the Figures, all this in very good agreement with the predictions of Fig. 1(b).

Fig. 3 illustrates the effect of increasing the input seed amplitude $A_{0}^{s}$ with all the remaining parameters fixed. The plots correspond to the propagation distance $\xi_{v}=0.75$ and corresponds to input conditions identical to those in Fig. 2, but now for different values of $A_{0}^{s}$. The location of the corresponding conditions in Fig. 1(b) is indicated with triangles. One observes that for the lowest amplitudes of the seed there are 4 vortices. The location of the two outer (a)

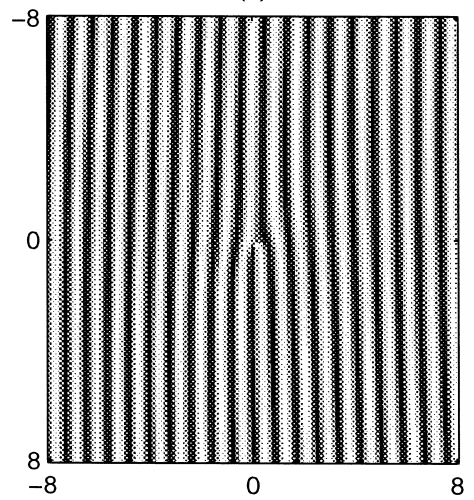

(b)

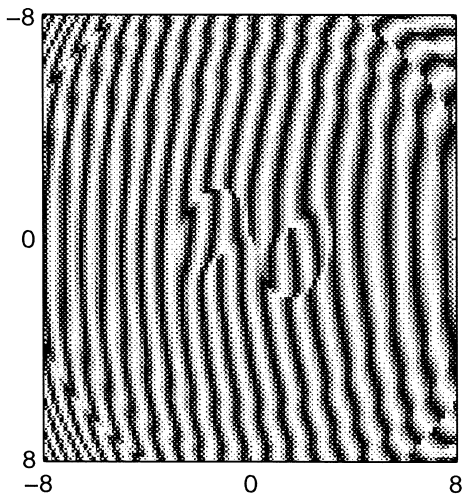

(c)

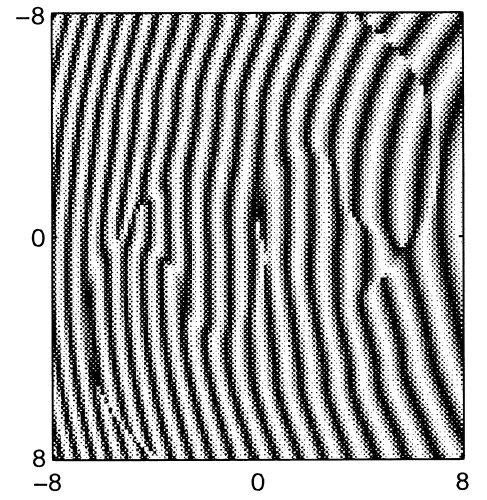

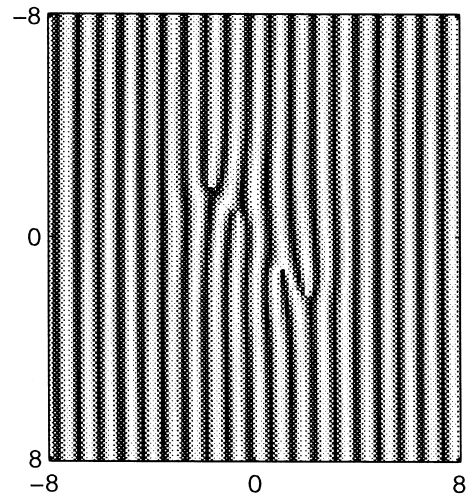
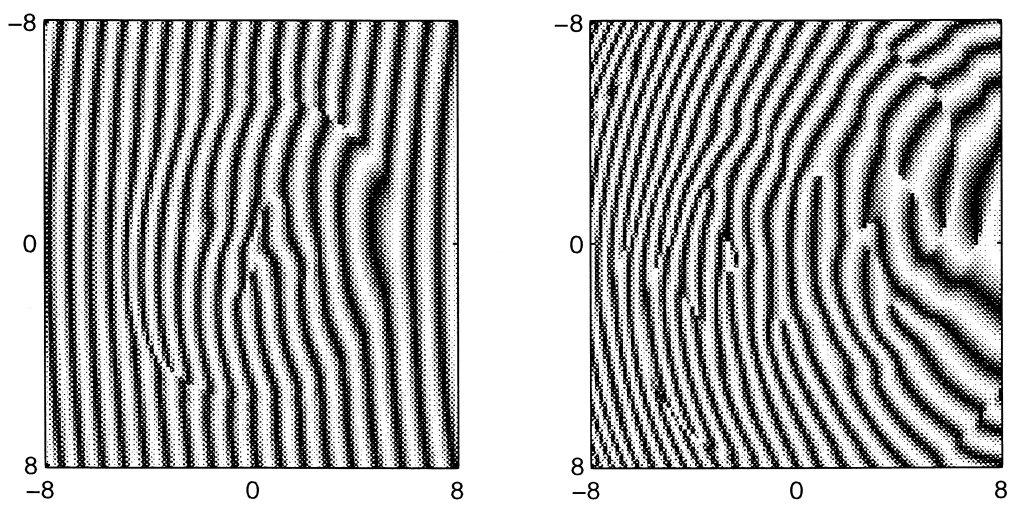

Fig. 5. Numerically obtained interferograms for both FF and SH beams for input conditions that do not fall under the low-depletion regime. Input conditions: $A_{0}=4, A_{0}^{s}=2, w_{0 v}=2, w_{o s}=2$, and $\beta=0$. Propagation distances: (a) $\xi=0.1$, (b) $\xi=1$, (c) $\xi=3$. Upper row: FF beam; lower row: $\mathrm{SH}$ beam. 
(a)

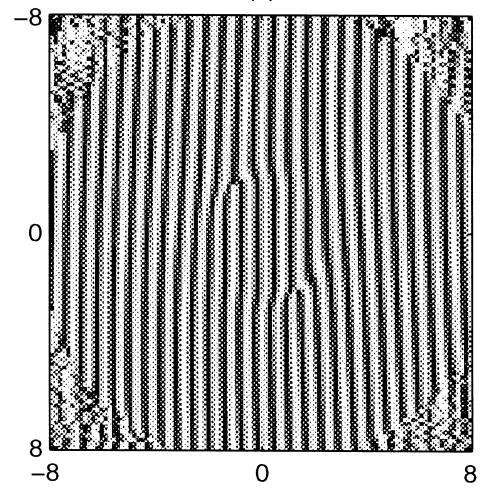

(c)

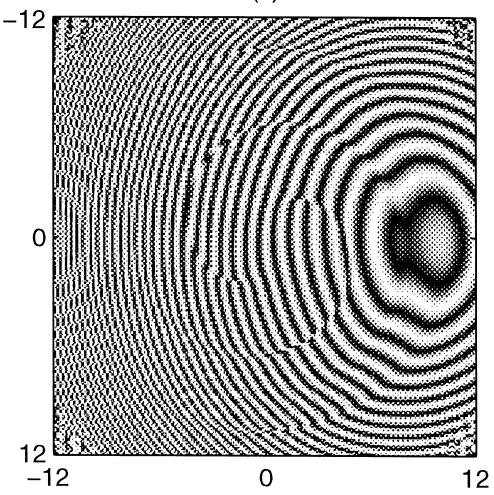

(b)

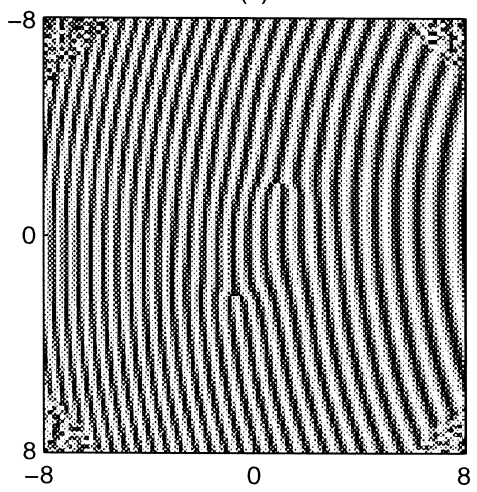

(d)

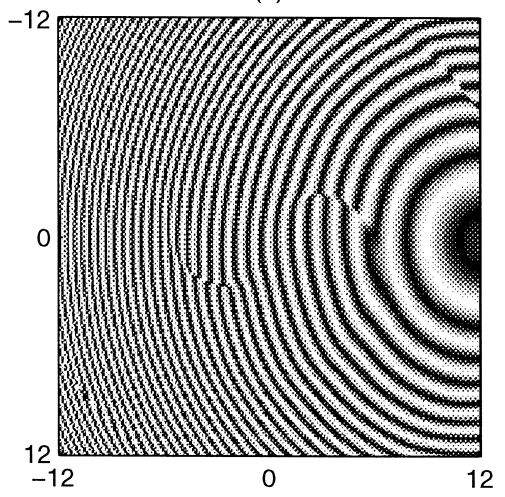

Fig. 6. Numerically obtained interferograms for the SH beam at different propagation distances inside the nonlinear crystal. Input conditions are: $A_{0}=0.2, A_{0}^{s}=0.08, w_{0 v}=2, w_{o s}=1$, thus $\kappa=0.5$, and $\beta=0$. Propagation distances: (a) $\xi=0.1$, (b) $\xi=1$, (c) $\xi=2$, (d) $\xi=3$.

ones shrunks towards the center when the seed amplitude increases and eventually they annihilate with the two other vortices when the ratio $A_{0}^{s} e / A_{0}^{2} z_{0 v}$ reaches the threshold value shown in Fig. 1(b).

Fig. 4 illustrates the features introduced by the presence of finite phase-mismatch. Conditions of the simulations are similar as in Fig. 2, except that here $\beta=5$. According to Fig. 1(b), in this case the region where there are four vortices in the wave front of the $\mathrm{SH}$ beam becomes very narrow. In particular, for the input conditions of Fig. 4 they exist only at an extremely narrow region of propagation distances, not displayed in the plot. However, input conditions can be chosen so that the 4 vortices are visible. The important conclusion raised by Fig. 1 and confirmed by Fig. 4 is that the most favorable conditions for the experimental observation of the 4 vortices occur at phasematching.

It is important to stress that Fig. 1 is only intended to hold under conditions of negligible depletion of the FF pump beam. Otherwise, its predictions are not necessarily valid. When the pump beam exhibits significant depletion power goes back and forth between the FF and the $\mathrm{SH}$ beams and new vortices can also appear in the FF beam.
Fig. 5 shows a typical example of such a complex dynamics. The location of the corresponding conditions in Fig. 1(b) is indicated with squares. The plots show interference

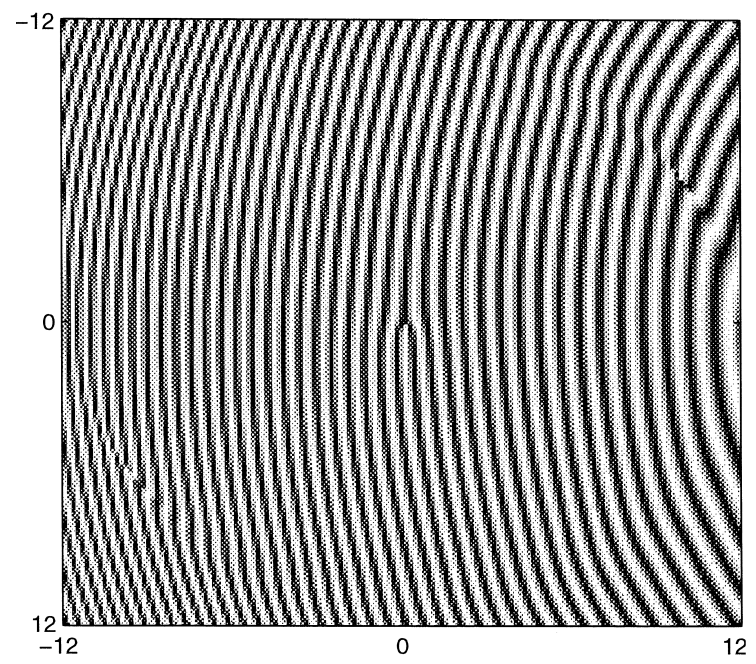

Fig. 7. Same as in Fig. 6(d), but for the FF beam. 
patterns of both FF and SH waves. Nucleation of new pairs of single-charge vortices of opposite charges in both the FF and the SH is clearly visible in the numerical simulation. Another new feature that is observed in the simulations is the spatial separation between the location of the vortices existing in the $\mathrm{FF}$ and the $\mathrm{SH}$ beams.

Because Fig. 5 corresponds to high-amplitude input beams, the drastic difference between the plots and the predictions of the negligible pump-depletion model are clearly visible. More subtle differences are obtained for lower-amplitude inputs. Fig. 6 illustrates such situation. It shows the interference patterns obtained for the $\mathrm{SH}$ beams when $\kappa=0.5$ under conditions where the very small, albeit not exactly vanishing, pump depletion. The location of the corresponding conditions in Fig. 1(a) is indicated with circles. One observes in the plots the single-charge vortices predicted by Fig. 1(a), namely 2 vortices at $\xi_{v}<$ $1 / \sqrt{2}$, and 4 vortices at $\xi_{v}>1 / \sqrt{2}$, plus 2 additional equally-charged single-charge vortices that appear far away from the beam center when $\xi_{v}>1 / \sqrt{2}$. A similar pair of equal-charge vortices appear in the FF beam, as shown in Fig. 7. To disregard the possibility that the additional
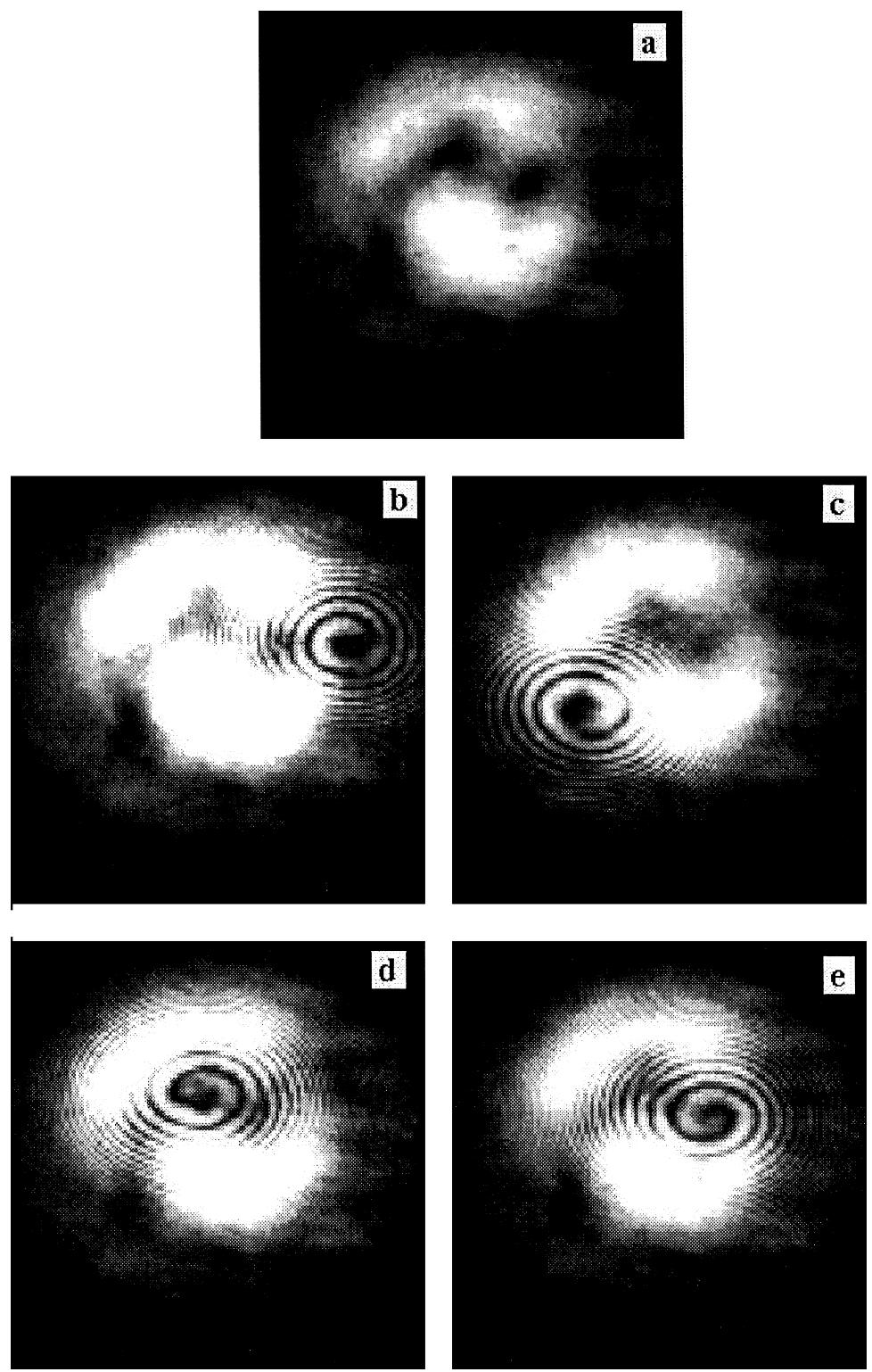

Fig. 8. Experimentally observed light distribution and interferograms at the SH wavelength $\lambda=0.532 \mathrm{~nm}$ measured at the output of the KTP crystal. See text for the actual values of the involved parameters. The interferograms were obtained by scanning the wave front of the output second-harmonic beam in different directions, and each picture corresponds to a different direction of the reference wave. 
vortices are numerically-induced, we repeated the simulations with different grid densities and widths and obtained identical results. In particular, when the nonlinearity was switched-off the vortices did not appear, consistent with the fact they are induced by the parametric wave interaction. The additional vortices always appear and stay at distances very far from the beam axis where the field amplitudes are very small. Therefore, they have a limited experimental relevance. Yet, they show the limitations of the negligible-pump depletion approximation in the rigorous mathematical sense. The detailed investigation of the fascinating dynamics of such depleted pump regime requires a separate investigation that falls far beyond the scope of this paper.

\section{Comparison with experiment}

Experimental observation of the nucleation of vortex pairs under conditions that fall inside the regime considered here was reported recently in Ref. [11]. Experiments were conducted in a $2 \mathrm{~cm}$ long potassium titanyl phosphate (KTP) crystal cut for Type II phase-matching for the pump fundamental wavelength $1.064 \mu \mathrm{m}$. A Q-switched $\mathrm{Nd}$ :YAG laser was used to provide coherent $8 \mathrm{~ns}$ pulses with a spatial Gaussian shape at the fundamental and second-harmonic frequencies. Both a pump FF beam with a charge 1 vortex dislocation and a weak vorticity-less $\mathrm{SH}$ seed were supplied at the entrance face of the crystal. Inside the accuracy of the experimental equipment, the widths of the beams were both equal to some $60 \mu \mathrm{m}$. Thus, the aspect ratio $\kappa \approx 1$, the diffraction length of the FF pump $z_{0 v} \approx 2 \mathrm{~cm}$, which coincides with the actual length of the crystal, and the critical length $\xi_{v} \approx 1$. The input light intensity was chosen to be well inside the low-depletion regime. Fig. 8 shows the experimentally observed light distribution and interferograms for the $\mathrm{SH}$ beam at the output of the crystal at phase-matching $(\beta=0)$ [11]. The pictures show the existence of two vortex pairs with zero net charge, consistent with the predictions of Fig. 1(b) for a wide range of input light intensities.

\section{Conclusions}

We presented a simple model to describe the evolution of vortex wave front dislocations in nonlinear parametric wave mixing in quadratic nonlinear crystals under conditions of low-depletion of the pump signal, and applied it to the case of seeded second-harmonic generation with pump beams with single charge vortices nested in Gaussian envelopes. In the framework of negligible pump-depletion regime the model predicts that the number of vortices in the SH beam may vary between 0,2 and 4, depending on the various beam parameters involved and on the propagation distance inside the nonlinear crystal. Predictions were shown to be in reasonable agreement with results of numerical simulations of the full governing equations and also consistent with previous experimental observations. The model can be potentially extended to general Laguerre-Gaussian input beam profiles $[13,14]$, to arbitrary topological charge mixing, to Type II phase-matching geometries, to include Poynting-vector walk-off, and to general three-wave mixing interactions. Of particular interest are the fascinating cases of vortex nucleation in quantum-noise parametric amplification [15], and the explosion of higher-order vortices [16] in walking parametric wave mixing.

\section{Acknowledgements}

This work was supported by the Spanish Government under contract PB95 0768 and through the Direccion General de Enseñanza Superior e Investigacion Cientifica.

\section{References}

[1] J.F. Nye, M.V. Berry, Proc. R. Soc. A 336 (1974) 165.

[2] M.V. Berry, Singularities in waves and rays, in: R. Balian, M. Kleman, J.-P. Poirier (Eds.), Physics of Defects, NorthHolland, Amsterdam, 1981.

[3] B. Luther-Davies, J. Christou, V. Tikhonenko, Y.S. Kivshar, J. Opt. Soc. Am. B 14 (1997) 3045.

[4] D. Rozas, C.T. Law, G.A. Swartzlander, J. Opt. Soc. Am. B 14 (1997) 3055.

[5] Y.S. Kivshar, B. Luther-Davies, Phys. Rep. 298 (1998) 81.

[6] M.S. Soskin, V.N. Gorshkov, M.V. Vasnetsov, J.T. Malos, N.R. Heckenberg, Phys. Rev. A 56 (1997) 4064.

[7] I.V. Basistiy, V.Y. Bazhenov, M.S. Soskin, M.V. Vasnetsov, Optics Comm. 103 (1993) 422.

[8] K. Dholakia, N.B. Simpson, M.J. Padgett, L. Allen, Phys. Rev. A 54 (1996) 3742.

[9] J. Courtial, K. Dholakia, L. Allen, M.J. Padgett, Phys. Rev. A 56 (1997) 4193.

[10] A. Berzanskis, A. Matijosius, A. Piskarskas, V. Smilgevicius, A. Stabinis, Optics Comm. 140 (1997) 273; 150 (1998) 372.

[11] D.V. Petrov, L. Torner, Phys. Rev. E 58 (1998) 7903.

[12] N.R. Heckenberg, R. McDuff, C.D. Smith, A.G. White, Optics Lett. 17 (1992) 221.

[13] A.E. Siegman, Lasers, U Science Books, Mill Valley, 1986.

[14] J. Courtial, Optics Comm. 151 (1998) 1.

[15] P.D. Trapani, A. Berzanskis, S. Minardi, S. Sapone, W. Chinaglia, Phys. Rev. Lett. 81 (1998) 5133.

[16] I. Freund, Optics Comm. 159 (1999) 99. 